\title{
On New Solutions of System of Shallow Water Wave Equations
}

\author{
Ziad Rached \\ Department of Mathematics \\ Faculty of Natural and Applied Sciences \\ Notre Dame University-Louaize \\ Zouk-Mosbeh, Lebanon \\ Email: zrached [AT] ndu.edu.lb
}

\begin{abstract}
Obtaining analytical solutions of nonlinear differential equations and nonlinear systems of partial and ordinary differential equations is an important topic in various fields of Mathematics. Many techniques are available in the literature. In this note, the enhanced modified simple equation method (EMSEM) is applied to system of shallow water wave equations.
\end{abstract}

Keywords--- Nonlinear system of differential equation, analytical solutions, EMSEM, shallow water wave equations

\section{INTRODUCTION}

Many methods are available for constructing analytical solutions of nonlinear equations and systems of equations: the Expfunction method [1,2], the tanh-function method [3,4], the homogeneous balance method [5,6], the $\left(G^{\prime} / G\right)$-expansion method [7,8], the Backlund transformation method [9], the Jacobi elliptic function method [10], the modified simple equation method [11,12], the enhanced modified simple equation method [13].

In the sequel, we obtain new analytical solutions of the system of shallow water wave equations

$$
\begin{array}{r}
u_{t}+u_{x} v+u v_{x}+v_{x x x}=0 \\
v_{t}+u_{x}+v v_{x}=0
\end{array}
$$

using the enhanced modified simple equation method.

In Section 2, the enhanced modified simple equation method is presented. In Section 3, the method is applied to the system of shallow water wave equations. Finally, discussion and concluding remarks are given in Section 4.

\section{THE ENHANCED MODIFIED SIMPLE EQUATION METHOD}

Any nonlinear partial differential equation is of the form

$$
F\left(u, u_{t}, u_{x}, u_{x x}, u_{x t}, u_{t t}, \ldots\right)=0(2)
$$

where $F$ is a polynomial of $u$ and its partial derivatives. The steps are as follows.

Step 1: Using the transformation

$$
u(x, t)=u(\zeta), \quad \zeta=p(t) x+q(t)
$$

where $p(t)$ and $q(t)$ are differentiable functions of $t$, from (2) and (3) we get the Ordinary differential equation

$$
F\left(u,\left(p^{\prime} x+q^{\prime}\right) u^{\prime}, p u^{\prime}, \ldots\right)=0
$$

Step 2: Assume (4) has solution

$$
u(\zeta)=\sum_{k=0}^{n} A_{k}(t)\left[\frac{\varphi^{\prime}(\zeta)}{\varphi(\zeta)}\right]^{k}
$$

where $A_{k}(t)$ and $\varphi(\zeta)$ are unknown expressions to be computed. It is assumed that $A_{n} \neq 0$. 
Step 3: Determine the positive integer $n$ in equation (5) by considering the homogeneous balance between the highest order derivatives and the nonlinear terms in equation (4).

Step 4: Substitute equation (5) into equation (4). Gather all the terms in the polynomial of the same power of $\varphi(\zeta)^{-j}$, where $j \geq 0$, and equate all the coefficients of these terms to zero. This yields a system of equations which can be solved to find $A_{k}(t)$ and $\varphi(\zeta)$. Consequently, we can get the exact solution of equation (2).

\section{APPLICATION TO SHALLOW WATER WAVE EQUATIONS}

In this section, we obtain exact solutions of shallow water wave equations

$$
\begin{aligned}
u_{t}+u_{x} v+u v_{x}+v_{x x x} & =0 \\
v_{t}+u_{x}+v v_{x} & =0
\end{aligned}
$$

Note that (3) reduces it into

$$
\begin{aligned}
& u^{\prime}\left(p^{\prime} x+q^{\prime}\right)+u^{\prime} p v+u v^{\prime} p+p^{3} v^{\prime \prime \prime}=0 \\
& v^{\prime}\left(p^{\prime} x+q^{\prime}\right)+u^{\prime} p+v v^{\prime} p=0
\end{aligned}
$$

Integrating (7) with respect to $\zeta$ yields

$$
v\left(p^{\prime} x+q^{\prime}\right)+u p+\frac{v^{2}}{2} p=0
$$

Hence

$u=-v\left(\frac{p^{\prime}}{p} x+\frac{q^{\prime}}{p}\right)-\frac{v^{2}}{2}$

From (7)

$$
u^{\prime}=-v v^{\prime}-\frac{v^{\prime}}{p}\left(p^{\prime} x+q^{\prime}\right)
$$

Replace $u$ and $u^{\prime}$ by their expressions given in (8) and (9) respectively in (6) yields

$$
-3 v v^{\prime}\left(p^{\prime} x+q^{\prime}\right)-\frac{v^{\prime}}{p}\left(p^{\prime} x+q^{\prime}\right)^{2}-\frac{3}{2} v^{2} v^{\prime} p+v^{\prime \prime \prime} p^{3}=0
$$

which, after integration with respect to $\zeta$ yields

$$
v^{\prime \prime} p^{3}-\frac{1}{2} p v^{3}-\frac{3}{2} v^{2}\left(p^{\prime} x+q^{\prime}\right)-\frac{v}{p}\left(p^{\prime} x+q^{\prime}\right)^{2}=0
$$

By balancing $v^{\prime \prime}$ and $v^{3}$, equation (10) has a solution of the following form:

$$
v(\zeta)=A_{0}(t)+A_{1}(t)\left[\frac{\varphi^{\prime}(\zeta)}{\varphi(\zeta)}\right]
$$

where $A_{0}(t)$ and $A_{1}(t)$ are expressions to be computed. It is assumed that $A_{1}(t) \neq 0$. We have

$$
\begin{aligned}
& v^{\prime}=A_{1}\left(\frac{\varphi^{\prime \prime}}{\varphi}-\left(\frac{\varphi^{\prime}}{\varphi}\right)^{2}\right) \\
& v^{\prime \prime}=A_{1}\left(\frac{\varphi^{\prime \prime \prime}}{\varphi}-\frac{3 \varphi^{\prime} \varphi^{\prime \prime}}{\varphi^{2}}+2\left(\frac{\varphi^{\prime}}{\varphi}\right)^{3}\right)
\end{aligned}
$$


Substituting equations (11)-(13) into equation (10) and equating all the coefficients of $\varphi^{0}, \varphi^{0} x, \varphi^{-1}, \varphi^{-1} x, \varphi^{-2}, \varphi^{-2} x$ and $\varphi^{-3}$ to zero, we get respectively

$$
\begin{aligned}
& \frac{p}{2} A_{0}^{3}+\frac{3}{2} A_{0}^{2} q^{\prime}+\frac{\left(q^{\prime}\right)^{2}}{p} A_{0}=0 \\
& \frac{3}{2} A_{0}^{2} p^{\prime}+\frac{2 p^{\prime} q^{\prime}}{p} A_{0}=0 \\
& A_{1} p^{3} \varphi^{\prime \prime \prime}-\frac{3}{2} p A_{1} A_{0}^{2} \varphi^{\prime}-3 A_{0} A_{1} \varphi^{\prime} q^{\prime}-\frac{\left(q^{\prime}\right)^{2}}{p} A_{1} \varphi^{\prime}=0 \\
& 3 A_{0} A_{1} p^{\prime} \varphi^{\prime}+\frac{2 p^{\prime} q^{\prime}}{p} A_{1} \varphi^{\prime}=0 \\
& 3 A_{1} p^{3} \varphi^{\prime} \varphi^{\prime \prime}+\frac{3}{2} p A_{0} A_{1}^{2}\left(\varphi^{\prime}\right)^{2}+\frac{3}{2} A_{0}^{2} A_{1}^{2}\left(\varphi^{\prime}\right)^{2} q^{\prime}=0 \\
& \frac{3}{2} p^{\prime} A_{1}^{2}\left(\varphi^{\prime}\right)^{2}=0 \\
& 2 A_{1} p^{3}\left(\varphi^{\prime}\right)^{3}-\frac{p}{2} A_{1}^{3}\left(\varphi^{\prime}\right)^{3}=0
\end{aligned}
$$

From (19), it follows that $p(t)=k$, where $k$ is some arbitrary constant different from zero. Note that from (18), $A_{0} \neq 0$, since otherwise, $A_{1}$ must be zero which contradicts the fact that $A_{1} \neq 0$.

From (14), it follows that

$$
k^{2} A_{0}^{2}+3 k A_{0} q^{\prime}+2\left(q^{\prime}\right)^{2}=0
$$

And hence

$$
A_{0}=\frac{-3 k q^{\prime} \pm \sqrt{q k^{2}\left(q^{\prime}\right)^{2}-8 k^{2}\left(q^{\prime}\right)^{2}}}{2 k^{2}}
$$

where $q(t)$ is an arbitrary function of $t$.

From (16)

$$
\varphi^{\prime \prime \prime}=M \varphi^{\prime}
$$

Where

$$
M=\left(\frac{\frac{3}{2} k A_{0}^{2}+3 A_{0} q^{\prime}-\frac{\left(q^{\prime}\right)^{2}}{k}}{k^{3}}\right)
$$

Equation (18) reduces to

$$
\varphi^{\prime}=N \varphi^{\prime \prime}
$$

Where

$N=-\frac{k^{3}}{\frac{k}{2} A_{0} A_{1}+\frac{A_{0}^{2}}{2} A_{1} q^{\prime}}$

where $A_{1}= \pm 2 k$ from (20) 
Substitute equation (25) into equation (23), we conclude that

$$
\frac{\varphi^{\prime \prime \prime}}{\varphi^{\prime \prime}}=M N
$$

Integrating equation (27) with respect to $\zeta$ yields

$$
\varphi^{\prime \prime}=c_{1} \exp [M N \zeta]
$$

Substituting equation (28) into equation (25), we conclude that

$$
\varphi^{\prime}=c_{1} N \exp [M N \zeta]
$$

Integrating equation (29) with respect to $\zeta$ yields

$$
\varphi=c_{2}+\frac{c_{1}}{M} \exp [M N \zeta]
$$

From (8), (11), (29), and (30) we get the following family of exact solutions of (1)

$$
\begin{gathered}
v(x, t)=A_{0}+A_{1}\left(\frac{c_{1} N \exp [M N \zeta]}{c_{2}+\frac{c_{1}}{M} \exp [M N \zeta]}\right) \\
u(x, t)=-v\left(\frac{q^{\prime}}{k}\right)-\frac{v^{2}}{2}
\end{gathered}
$$

\section{CONCLUSION}

The enhanced modified simple equation method is applied successfully to the system of shallow water wave equations. New solutions are obtained.

\section{REFERENCES}

[1] J. H. He and X. H. Wu, "Exp-function method for nonlinear wave equations," Chaos, Solitons \& Fractals, vol. 30, no. 3, pp. 700-708, 2006.

[2] R. Sakthivel, and C. Chun, "New Soliton Solutions of Chafee-Infante Equations using the Exp-Function Method," Z. Naturforsch, 65a, pp. 197-202, 2010.

[3] M. A. Abdou, "The extended Tanh method and its applications for solving nonlinear physical models," Applied Mathematics and Computation, vol. 190, no. 1, pp. 988-996, 2007.

[4] E. Fan, "Extended tanh-function method and its applications to nonlinear equations," Physics Letters A, vol. 277, no. 4-5, pp. 212-218, 2000.

[5] M. L. Wang, “Solitary wave solutions for variant Boussinesq equations,” Physics Letters A, vol. 199, no. 3-4, pp. 169$172,1995$.

[6] E. M. E. Zayed, H. A. Zedan, and K. A. Gepreel, "On the solitary wave solutions for nonlinear Hirota-Satsuma coupled KdV of equations,” Chaos, Solitons \& Fractals, vol. 22, no. 2, pp. 285-303,2004.

[7] M. Wang, X. Li, and J. Zhang, "The (G'/G)-expansion method and travelling wave solutions of nonlinear evolution equations in mathematical physics," Physics Letters A, vol. 372, no. 4, pp. 417-423, 2008.

[8] E. Aksoy, A. Bekir, "Exact solutions of shallow water wave equations by using the (G'/G)-expansion method," waves in random and complex media, 22 (3), pp. 1-15, 2012.

[9] M. R. Miura, Backlund Transformation, Springer, Berlin, Germany, 1978.

[10] D. Lu and Q. Shi, "New Jacobi elliptic functions solutions for the combined KdV-MKdV equation," International Journal of Nonlinear science, vol. 10, no. 3, pp. 320-325, 2010. 
[11] A. J. M. Jawad, M. D. Petkovic', and A. Biswas, "Modified simple equation method for nonlinear evolution equations," Applied Mathematics and Computation, vol. 217, no. 2, pp. 869-877, 2010.

[12] E. M. E. Zayed and S. A. H. Ibrahim, "Exact solutions of nonlinear evolution equations in mathematical physics using the modified simple equation method," Chinese Physics Letters, vol. 29, no. 6, Article ID 060201, 2012.

[13] C. Zhang and Z. Zhang, "Application of the enhanced modified simple equation method for Burger-Fisher and modified Volterra equations," Advances in Difference Equations, 2017 